\title{
Role of Alpha Hemoglobin Stabilizing Protein Expression in Beta Thalassemia Patients
}

\author{
IMAN A. AHMEDY, M.D.*; SAMIA H. KANDEL, M.D.*; SAFAA I. TAYEL, M.D.** and \\ MAHMOUD A. EL-HAWY, M.D.*** \\ The Departments of Clinical Pathology*, Medical Biochemistry \& Molecular Biology** and Pediatrics***, \\ Faculty of Medicine, Menoufia University, Menoufia, Egypt
}

\begin{abstract}
Background: Beta $(\beta)$-Thalassemia is an autosomal recessive inherited disease causing variable degrees of anemia. The molecular defects are owing to point mutations or small deletions leading to diminution or absence of $\beta$-globin chain synthesis. The unmatched $\alpha-\mathrm{Hb}$ is destructive to itself and other cellular components, resulting in ineffective erythropoiesis and hemolysis. Alpha-Hemoglobin Stabilizing Protein (AHSP) is a scavenger protein of erythroid that fastly and reversibly attach the $\alpha$ subunit of hemoglobin molecule preventing its precipitation and subsequent hemolysis.
\end{abstract}

Aim of Work: Is to assess AHSP gene expression in thalassemia major and intermedia and its relation to clinical features, laboratory investigations and to determine its role on the severity of thalassemia.

Subjects and Methods: This case-control study is consisted of 120 studied subjects categorized into 40 thalassemia major patients, 40 thalassemia intermedia patients and 40 age healthy individuals as a control group. All subjects underwent the following; history, clinical picture, complete blood picture and $\mathrm{Hb}$ electrophoresis, serum ferritin and measurement of AHSP, alpha globin and beta globin genes expression by real time PCR.

Results: AHSP gene in thal. intermedia group was higher than both thal. major $(p<0.001)$. Beside thal. intermedia group had higher $\alpha$ globin/AHSP ratio versus thal. major group $(p=0.011)$. $\beta$ globin/AHSP ratio was not significant between both thal. groups. AHSP in thal.major group correlated with $\alpha$ globin and $\beta$ globin expression and negatively correlated with organomegally, ferritin and no. of blood transfusion. Whereas in thal. intermedia group, was positively correlated with $\mathrm{Hb}, \mathrm{HCT}, \mathrm{MCV}, \mathrm{MCH}, \alpha$ globin gene expression, $\beta$ globin gene expression and $\beta$ globin $/ \alpha$ globin genes ratio.

Conclusion: AHSP expression was higher in thalassemia intermedia group than thalassemia major group. Consequently, AHSP can be considered genetic modifier in $\beta$ thalassemia.

Key Words: AHSP - Thalassemia intermedia-Thalassemia major - Flowcytometry.

Correspondence to: Dr. Iman A. Ahmedy, E-Mail: imanahmedy@yahoo.com

\section{Introduction}

THALASSEMIA is an inherited world wide blood disorder that results in formation of abnormal hemoglobin [1]. It is the most widespread hemolytic anemia in Egypt with carrier rates ranging from 9 to $16 \%$, constituting $45 \%$ of the total hematological patients and $86 \%$ of chronic hemolytic pediatric patients [2]

The molecular defects are owing to point mutations or small deletions within the chromosome $11 \beta$-globin gene leading to diminution or absence of the synthesis of $\beta$-globin chain. The unmatched $\alpha-\mathrm{Hb}$ is destructive to itself and other cellular components due to its oxidative instability [3] ensuing by hemolysis of circulating RBCs and also impaired erythroid precursors viability in hematopoietic tissues [4]

Alpha-Hemoglobin Stabilizing Protein (AHSP) is an erythroid scavenger protein that quickly and reversibly binds to $\alpha$ subunit monomeric forms. It also enhance modulation of oxidation of heme iron and subunit folding [5]

AHSP, known as an alpha-hemoglobin $(\alpha-\mathrm{Hb})$ chaperone which specifically recognizes the of $\alpha$ $\mathrm{Hb} \mathrm{G}$ and $\mathrm{H}$ helices, forming a steady complex with free $\alpha-\mathrm{Hb}$ till it bind its $\beta$-subunits partner. Unlike the soluble free $\beta-\mathrm{Hb}$ which forms homologous tetramers, newly synthesized $\alpha-\mathrm{Hb}$ chains are highly unstable molecules which precipitate and create reactive oxygen species inside the erythrocyte precursors of the bone marrow inducing ineffective erythropoiesis and apoptosis [6]. Inspite of linking AHSP expression to the severity of beta thalassemia, its function as a potential genetic modifier of disease severity, has still not been clearly recognized [7]. Consecutively we aimed to 
investigate AHSP gene expression in thalassemia major and intermedia and its relation with alpha and beta globin genes expression and with clinical features and severity of thalassemia.

\section{Material and Methods}

This case control study was conducted in Clinical Pathology Departments in collaboration with Medical Biochemistry, Molecular Biology and Pediatric Departments, Faculty of Medicine, Menoufia University. In a period between 1/2016 to 11/2017. It included 120 studied subjects that were categorized into the following groups: Group I: Included 40 thalassemia major patients with age mean $(7.99 \pm 4.27)$. They were $23(57.5 \%)$ males and 17 (42.5\%) females. Group II: Included 40 thalassemia intermedia. Patients with age mean (8.82 \pm 4.48$)$. They were $23(57.5 \%)$ males and 17 (42.5\%) females Group III: Included 40 age and gender matched apparently healthy individuals as a control group with age mean $(6.87 \pm 3.75)$. They were $22(55 \%)$ males and $18(45 \%)$ females. Thalassemia patients were recruited from Pediatric Department, Menoufia University Hospitals and were documented by clinical picture, blood picture and $\mathrm{Hb}$ electrophoresis.

An informed written consent was retrieved from parents or care-givers before enrollment. The study was agreed on by the Ethical Committee of Medical Research, Faculty of Medicine, Menoufia University. It was conducted according to Helsinki Declaration. All patients underwent the following: Full history taking, general examination and laboratory investigations. Exclusion criteria included patients with any other haemoglobinpathies or haematological disorder, patients or families who refused or discontinued the study and patients with inflammation.

\section{Blood sampling and laboratory investigations:}

Venous blood was taken on EDTA tubes. The following investigations were done; complete blood count (Sysmex XN-1000, Japan (19723), B.M Egypt Company) and $\mathrm{Hb}$ electrophoresis (automated analyzer (minilite) MNL320350-Italy). AHSP, alpha globin and beta globin genes expression by quantitative real time Polymerase Chain Reaction (qPCR). Moreover, serum ferritin was estimated by Enzyme Linked Immunosorbent Assay (ELISA).

Measurement of AHSP, alpha globin and beta globin genes expression:

Quantitative assay of AHSP, alpha globin and beta globin genes expression in whole blood using Reverse Transcriptase Polymerase Chain Reaction
(RT-PCR) technique were done as follow: Total RNA isolation from whole blood using Kits supplied by (PureLink® RNA Mini Kit, Life Technologies, Carlsbad, California, USA), followed by assuring RNA quality and purity. Extracted RNA was stored in $-80^{\circ} \mathrm{C}$ till time of use. First stepPCR was cDNA synthesis (reverse transcription step) using (cDNA reverse transcription kits, Applied Biosystem), using Applied Bio systems 2720 thermal cycler (Singapore). Second step-PCR, was cDNA amplification (real time PCR step) Fig. (1): The cDNA was used in SYBR green based quantitative real-time PCR for quantification of by (SensiFAST TM SYBR Lo-ROX Kit from USA), using the following designed primers (Thermofisher Scientific, Invitrogen, USA). Alpha globin gene primers:

Forward primer 5 AACTTCAAGCTCC TAAGCCACTGC-3', Reverse primer 5'- CGAGGCTCCAGCTTAACGGTATTTG -3` and Beta globin primer sequence: Forward primer 5'ATCCTGAGAACTTCAGGCTCCTGGG - 3', Reverse primer 5 GAGCTTAGTGATACTTGTGGGCCAG-3 and AHSP Forward primer 5'CCTGTTAGACCTGAAGGCAGATGGC-3’, Reverse primer 5 -AGTCCTCCACCACAGTCACCA TGT-3 and Beta actin Forward primer 5 CCACACTGTGCCCATCTACG-3` Reverse primer 5`-CCGTGGTGGTGAAGCTGTAG.

Applied Biosystems 7500 software Version 2.0.1 was used for data analysis Relative Quantification (RQ) of gene expression was performed using comparative $\Delta \Delta \mathrm{Ct}$ method [8]. The target mRNA, is normalized to an endogenous reference gene (Beta actin) and relative to a control. Each run was accomplished using melting curve analysis Fig. (2) to confirm amplification specificity and primer dimers absence.

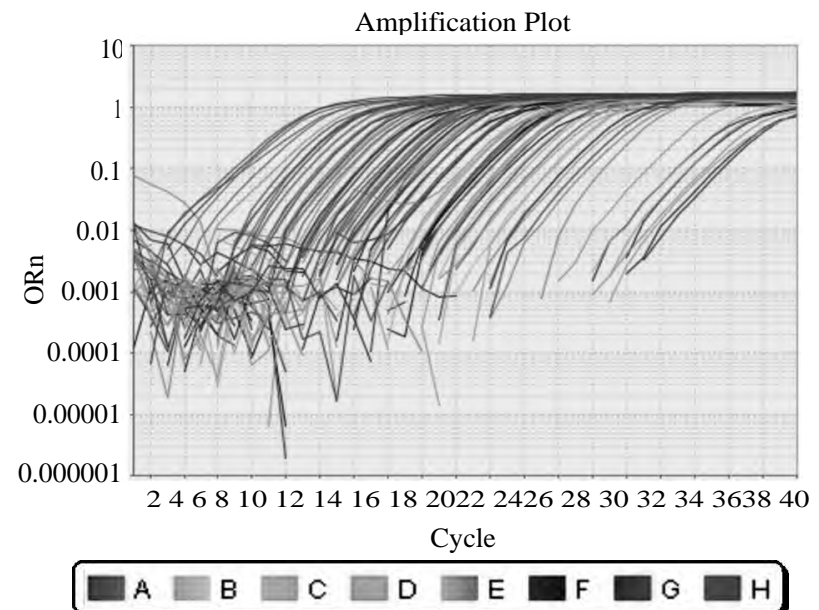

Fig. (1): Amplification plot of AHSP, alpha globin and beta globin genes expression (normalized fluorescence signal $(\Delta \mathrm{Rn})$ plotted versus cycle number). 


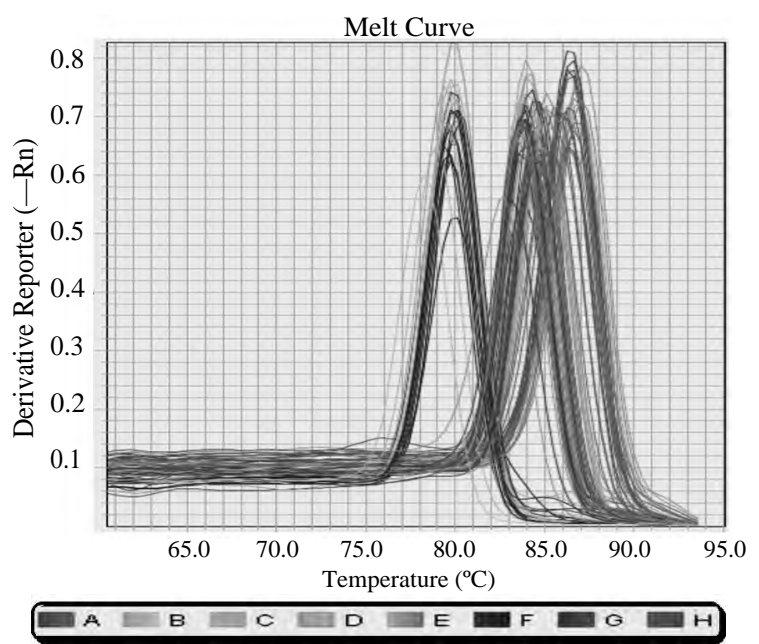

Fig. (2): Melting curve analysis of AHSP, alpha globin and beta globin genes expression (derivative reporter $(-\mathrm{Rn})$ plotted versus temperature).

Statistical analysis:

Data were fed to the computer and analyzed using IBM SPSS software package Version 20.0 (Armonk, NY: IBM Corp).

\section{Results}

The current study depicted no significant statistical difference in between the studied groups as regard age \& sex (Table 1). Moreover, at the clinical level, a statistical difference between thal. major versus thal. intermedia and control cases $(p<0.001)$ as regard all clinical features were found (Table 2). On the other hand there was a highly statistical difference between thal. intermedia and control group as regard pallor $(p<0.001) \&$ jaundice $(p \leq$ 0.05 ). While no statistical difference was found with organomegally. Regarding hematological parameters, they were all lower in both thal. groups versus control group $(p<0.001)$ except RDW which was higher in both thalassemia groups versus control group $(p<0.001)$. In between both thalassemia group, all hematological parameters were lower in thal. major than thal. intermedia ( $p<0.001), \operatorname{MCV}(p<$ 0.05 ) except RDW \& MCH which didn't show any statistical difference between both groups (Table 3).

Moreover $\mathrm{HbF} \%$ and ferritin were significantly different among groups $(p<0.001)$. HbF mean \pm SD was $21.11 \pm 26.75,43.74 \pm 23.62$ and $21 \pm 0.21$ in thal. major, thal. intermediate and control group respectively $(p<0.001)$. $\mathrm{HbF} \%$ was found to be higher in both thalassemia groups than control group $(p<0.001)$. In between thalassemia groups it was higher in thal. intermedia group than thal. major group $(p<0.001)$ (Table 3$)$.

On the other hand ferritin mean \pm SD was $2140.7 \pm 1144.4,245.4 \pm 135.4$ and $48.33 \pm 15.71$ in thal. major, thal. intermediate and control group respectively $(p<0.001)$. It was higher in thalassemia groups than control $(p<0.001)$. Moreover, it was higher in thal. major than thal. intermediate ( $p$ $<0.001$ ) (Table 3).

Furthermore, the frequency of blood transfusion was significantly higher in thal. major group (mean $\pm \mathrm{SD}=9.15 \pm 3.44)$ compared with thal. intermedia group (mean $\pm \mathrm{SD}=2.60 \pm 0.74)(p<0.001)$ (Table 3$)$.

Concerning globin gene expression, $\alpha$ globin gene expression was higher in thal. intermedia group versus thal. major $(p<0.001)$ and control groups $(p<0.05)$. While $\beta$ globin gene expression was lower in thal. major group compared with thal. intermedia $(p<0.05)$ and control groups $(p<0.001) . \beta$ globin $/ \alpha$ globin genes ratio was higher in control group compared with both thal. group $(p<0.001)$, while it did not differ between thal. major and thal. intermedia groups (Table 4). Apropos AHSP gene, thal. intermedia group expressed higher level versus both thal. major and control groups $(p<0.001)$, while, it was higher in thal. major group versus control group $(p=0.008)$. On the other hand, $\alpha$ globin/AHSP ratio was higher in control group versus both thal. groups $(p<0.001)$ (Table 4). Beside thal. intermedia group had higher $\alpha$ globin/AHSP ratio versus thal. major group ( $p=$ $0.011)$. While $\beta$ globin/AHSP ratio, was higher in control group versus both thal. groups $(p<0.001)$, while it was not significant between both thal. groups (Table 4). Spearman coefficient revealed that AHSP expression in thal. major group was positively associated with $\alpha$ globin and $\beta$ globin gene expression and negatively associated with organomegally, ferritin and no. of blood transfusion (Table 5). Whereas in thal. intermedia group, it was positively correlated with $\mathrm{Hb}, \mathrm{HCT}, \mathrm{MCV}$, $\mathrm{MCH}, \alpha$ globin gene expression, $\beta$ globin gene expression and $\beta$ globin/ $\alpha$ globin genes ratio (Table 5 ). Multivariate regression analysis depicted that organomegally, $\alpha$ globin and $\beta$ globin expression are the most independent factors affecting AHSP expression in thal. major group on the other hand (Table 6), $\alpha$ globin and $\beta$ globin genes expression are the most independent factors affecting AHSP expression in thal. intermedia group (Table 7). Receiver Operating Characteristic (ROC curve) showed that AHSP expression at cutoff $>1$ can discriminate thalassemia cases from healthy control with sensitivity $76.25 \%$, specificity $80 \%$ Fig. (3), whereas AHSP expression at cutoff $\leq 11.31$ can discriminate thalassemia major from thalassemia intermedia with sensitivity $(92.5 \%)$, specificity (60.0\%) Fig. (3). 


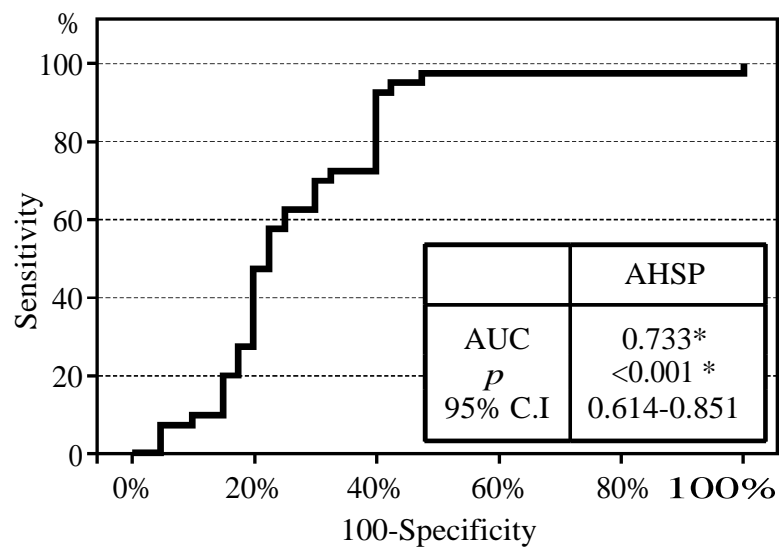

Fig. (3): ROC curve for AHSP to predict cases from control.

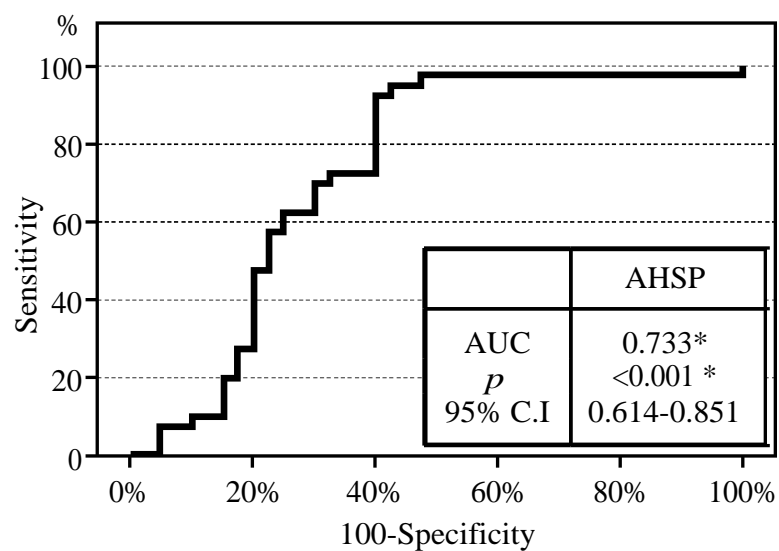

Fig. (4): Roc curve for AHSP to predict thalassemia major from intermedia.

Table (1): Comparison among the three studied groups regarding demographic data.

\begin{tabular}{llllll}
\hline & \multicolumn{1}{c}{$\begin{array}{c}\text { Major } \\
(\mathrm{n}=40)\end{array}$} & \multicolumn{1}{c}{$\begin{array}{c}\text { Interm } \\
(\mathrm{n}=40)\end{array}$} & $\begin{array}{l}\text { Control } \\
(\mathrm{n}=40)\end{array}$ & Test of sig. & $p$ \\
\hline Age (years): & & & & & \\
Min.-Max. & $1.50-17.0$ & $0.75-17.0$ & $0.50-15.0$ & $\mathrm{~K}=$ & 0.131 \\
Mean \pm SD. & $7.99 \pm 4.27$ & $8.82 \pm 4.48$ & $6.87 \pm 3.75$ & 4.064 & \\
Median & 8.0 & 9.0 & 6.5 & & \\
Sex (No \%): & & & & & \\
Males & $23(57.5 \%)$ & $23(57.5 \%)$ & $22(55.0 \%)$ & $\chi^{2}=$ & 0.967 \\
Females & $17(42.5 \%)$ & $17(42.5 \%)$ & $18(45.0 \%)$ & 0.068 & \\
\hline
\end{tabular}

Table (2): Comparison of the three studied groups regarding clinical features of thalassemia.

\begin{tabular}{|c|c|c|c|c|c|c|c|c|c|c|c|}
\hline & \multirow{2}{*}{\multicolumn{2}{|c|}{$\begin{array}{l}\text { Major } \\
(\mathrm{n}=40)\end{array}$}} & \multirow{2}{*}{\multicolumn{2}{|c|}{$\begin{array}{l}\text { Interm } \\
(\mathrm{n}=40)\end{array}$}} & \multirow{2}{*}{\multicolumn{2}{|c|}{$\begin{array}{l}\text { Control } \\
(n=40)\end{array}$}} & \multirow{3}{*}{$\chi^{2}$} & \multirow{3}{*}{$p$} & \multicolumn{3}{|c|}{ Sig. bet. grps } \\
\hline & & & & & & & & & \multirow{2}{*}{$\begin{array}{c}\text { Major } \\
\text { vs. interm }\end{array}$} & \multirow{2}{*}{$\begin{array}{c}\text { Major } \\
\text { vs. control }\end{array}$} & \multirow{2}{*}{$\begin{array}{c}\text { Interm } \\
\text { vs. control }\end{array}$} \\
\hline & No. & $\%$ & No. & $\%$ & No. & $\%$ & & & & & \\
\hline \multicolumn{12}{|l|}{ Pallor: } \\
\hline Negative & 0 & 0.0 & 0 & 0.0 & 40 & 100.0 & 120.000 & $<0.001 *$ & & $<0.001 *$ & $<0.001 *$ \\
\hline Positive & 40 & 100.0 & 40 & 100.0 & 0 & 0.0 & & & & & \\
\hline \multicolumn{12}{|l|}{ Jaundice: } \\
\hline Negative & 5 & 12.5 & 32 & 80.0 & 40 & 100.0 & $73.138^{*}$ & $<0.001 *$ & $<0.001 *$ & $<0.001 *$ & $0.005^{*}$ \\
\hline Positive & 35 & 87.5 & 8 & 20.0 & 0 & 0.0 & & & & & \\
\hline \multicolumn{12}{|c|}{ Organomegally: } \\
\hline Negative & 7 & 17.5 & 35 & 87.5 & 40 & 100.0 & $73.094 *$ & $<0.001 *$ & $<0.001 *$ & $<0.001 *$ & 0.055 \\
\hline Positive & 33 & 82.5 & 5 & 12.5 & 0 & 0.0 & & & & & \\
\hline \multicolumn{12}{|c|}{ Growth retardation: } \\
\hline Negative & 1 & 2.5 & 40 & 100.0 & 40 & 100.0 & $115.556^{*}$ & $<0.001 *$ & $<0.001 *$ & $<0.001 *$ & - \\
\hline Positive & 39 & 97.5 & 0 & 0.0 & 0 & 0.0 & & & & & \\
\hline \multicolumn{12}{|l|}{ Facies: } \\
\hline Negative & 16 & 40.0 & 40 & 100.0 & 40 & 100.0 & $60.000 *$ & $<0.001 *$ & $<0.001 *$ & $<0.001 *$ & - \\
\hline Positive & 24 & 60.0 & 0 & 0.0 & 0 & 0.0 & & & & & \\
\hline \multicolumn{12}{|l|}{ Splenectomy: } \\
\hline Negative & 32 & 80.0 & 40 & 100.0 & - & - & $8.889^{*}$ & $\mathrm{FE}_{p}=$ & - & - & - \\
\hline Positive & 8 & 20.0 & 0 & 0.0 & - & - & & $0.005^{*}$ & & & \\
\hline
\end{tabular}

$\chi 2, p: \chi^{2}$ and $p$-values for Chi square test for comparing among the three groups.

$\mathrm{FE}_{p}: p$-value for Fisher Exact for Chi square test for comparing between the two groups. : Statistically significant at $p \leq 0.05$. 
Table (3): Comparison among the three studied groups regarding laboratory data.

\begin{tabular}{|c|c|c|c|c|c|c|c|c|}
\hline & \multirow{2}{*}{$\begin{array}{l}\text { Major } \\
(n=40)\end{array}$} & \multirow{2}{*}{$\begin{array}{l}\text { Interm } \\
(n=40)\end{array}$} & \multirow{2}{*}{$\begin{array}{c}\text { Control } \\
(\mathrm{n}=40)\end{array}$} & \multirow{2}{*}{$\begin{array}{l}\text { Test of } \\
\text { sig. }\end{array}$} & \multirow[b]{2}{*}{$p$} & \multicolumn{3}{|c|}{ Post hoc test (LSD or Dunn's) } \\
\hline & & & & & & $\begin{array}{c}\text { Major } \\
\text { vs. interm }\end{array}$ & $\begin{array}{c}\text { Major } \\
\text { vs. control }\end{array}$ & $\begin{array}{c}\text { Interm } \\
\text { vs. control }\end{array}$ \\
\hline \multicolumn{9}{|c|}{$\operatorname{RBCs}\left(\times 10^{3} / \mathrm{e}\right) \cdot \mathrm{e}$} \\
\hline Min.-Max. & $1.64-3.88$ & $2.27-5.03$ & $4.14-5.63$ & $\mathrm{~F}=$ & $<0.001 *$ & $<0.001 *$ & $<0.001 *$ & $<0.001 *$ \\
\hline Mean \pm SD & $2.73 \pm 0.48$ & $3.22 \pm 0.74$ & $4.84 \pm 0.41$ & $154.837 *$ & & & & \\
\hline Median & 2.66 & 3.0 & 4.77 & & & & & \\
\hline \multicolumn{9}{|l|}{$H B(g / d l):$} \\
\hline Min.-Max. & $5.0-9.60$ & $5.50-11.50$ & $9.90-13.90$ & $\mathrm{~F}=$ & $<0.001^{*}$ & $<0.001 *$ & $<0.001 *$ & $<0.001 *$ \\
\hline Mean \pm SD & $7.10 \pm 1.14$ & $8.54 \pm 1.46$ & $11.77 \pm 0.99$ & $155.216^{*}$ & & & & \\
\hline Median & 7.0 & 8.50 & 11.50 & & & & & \\
\hline \multicolumn{9}{|l|}{ HCT: } \\
\hline Min.-Max. & $15.0-28.10$ & $15.10-34.60$ & $32.0-40.0$ & $\mathrm{~F}=$ & $<0.001^{*}$ & $<0.001 *$ & $<0.001 *$ & $<0.001 *$ \\
\hline Mean \pm SD. & $19.97 \pm 3.47$ & $25.22 \pm 4.87$ & $35.46 \pm 2.50$ & $177.195^{*}$ & & & & \\
\hline Median & 19.25 & 25.15 & 35.25 & & & & & \\
\hline \multicolumn{9}{|l|}{$M C V:$} \\
\hline Min.-Max. & $63.0-83.0$ & $63.40-79.0$ & $78.0-100.0$ & $\mathrm{~F}=$ & $<0.001^{*}$ & $0.034 *$ & $<0.001 *$ & $<0.001 *$ \\
\hline Mean \pm SD. & $71.59 \pm 5.01$ & $74.0 \pm 4.20$ & $88.20 \pm 5.77$ & $127.150 *$ & & & & \\
\hline Median & 72.60 & 75.40 & 88.0 & & & & & \\
\hline \multicolumn{9}{|l|}{$M C H:$} \\
\hline Min.-Max. & $20.70-29.0$ & $21.30-31.0$ & $29.0-33.0$ & $\mathrm{~F}=$ & $<0.001^{*}$ & 0.175 & $<0.001 *$ & $<0.001 *$ \\
\hline Mean \pm SD. & $25.52 \pm 2.09$ & $26.07 \pm 1.90$ & $31.07 \pm 1.33$ & 115.442 & & & & \\
\hline Median & 25.70 & 26.0 & 31.0 & & & & & \\
\hline \multicolumn{9}{|l|}{$R D W:$} \\
\hline Min.-Max. & $12.90-27.50$ & $13.50-28.0$ & $11.50-14.50$ & $\mathrm{~K}=$ & $<0.001^{*}$ & 0.376 & $<0.001 *$ & $<0.001 *$ \\
\hline Mean \pm SD & $19.73 \pm 3.77$ & $19.08 \pm 3.06$ & $13.49 \pm 0.93$ & $70.431 *$ & & & & \\
\hline Median & 20.20 & 19.0 & 14.0 & & & & & \\
\hline \multicolumn{9}{|l|}{ HbF\%: } \\
\hline Min.-Max. & $0.0-91.0$ & $0.0-96.80$ & $0.0-0.80$ & $\mathrm{~K}=$ & $<0.001^{*}$ & $0.001 *$ & $<0.001 *$ & $<0.001 *$ \\
\hline Mean \pm SD & $21.11 \pm 26.75$ & $43.74 \pm 23.62$ & $0.21 \pm 0.21$ & $68.090 *$ & & & & \\
\hline Median & 10.40 & 43.20 & 0.10 & & & & & \\
\hline \multicolumn{9}{|l|}{ Ferritin: } \\
\hline Min.-Max. & $71.40-5340.0$ & $86.0-851.0$ & $30.0-88.0$ & $\mathrm{~K}=$ & $<0.001 *$ & $<0.001 *$ & $<0.001 *$ & $<0.001 *$ \\
\hline Mean \pm SD. & $2140.7 \pm 1144.4$ & $245.4 \pm 135.4$ & $48.33 \pm 15.71$ & $101.331 *$ & & & & \\
\hline Median & 1803.5 & 210.50 & 42.65 & & & & & \\
\hline \multicolumn{9}{|c|}{ Frequency of blood } \\
\hline Min.-Max. & $2.0-14.0$ & $1.0-4.0$ & - & $\mathrm{U}=$ & $<0.001^{*}$ & & & \\
\hline Mean \pm SD. & $9.15 \pm 3.44$ & $2.60 \pm 0.74$ & - & $49.0 *$ & & & & \\
\hline Median & 9.50 & 3.0 & - & & & & & \\
\hline
\end{tabular}

F, $p:$ F and $p$-values for ANOVA test, Sig. bet. grps was done using Post Hoc Test (LSD).

$\mathrm{K}, p: \mathrm{K}$ and $p$-values for Kruskal Wallis test, Sig. bet. grps was done using Post Hoc Test (Dunn's multiple comparisons test).

$\chi^{2}, p: \chi 2$ and $p$-values for Chi square test for comparing between the three groups $\mathrm{U}$.

$p_{*} \quad: \quad \mathrm{U}$ and $p$-values for Mann Whitney test for comparing between the two groups. Statistically significant at $p \leq 0.05$. 
Table (4): Comparison among the three studied groups regarding $\alpha$ globin, B globin and AHSP genes expression.

\begin{tabular}{|c|c|c|c|c|c|c|c|c|}
\hline & \multirow{2}{*}{$\begin{array}{l}\text { Major } \\
(\mathrm{n}=40)\end{array}$} & \multirow{2}{*}{$\begin{array}{l}\text { Interm } \\
(\mathrm{n}=40)\end{array}$} & \multirow{2}{*}{$\begin{array}{c}\text { Control } \\
(\mathrm{n}=40)\end{array}$} & \multirow[b]{2}{*}{$\mathrm{K}$} & \multirow[b]{2}{*}{$p$} & \multicolumn{3}{|c|}{ Post hoc test (Dunn's) } \\
\hline & & & & & & $\begin{array}{c}\text { Major } \\
\text { vs. interm }\end{array}$ & $\begin{array}{c}\text { Major } \\
\text { vs. control }\end{array}$ & $\begin{array}{c}\text { Interm } \\
\text { vs. control }\end{array}$ \\
\hline $\begin{array}{l}\alpha \text { globin: } \\
\text { Min.-Max. } \\
\text { Mean } \pm \text { SD. } \\
\text { Median }\end{array}$ & $\begin{array}{l}0.0-6.41 \\
1.10 \pm 1.59 \\
0.42\end{array}$ & $\begin{array}{l}0.01-33.79 \\
9.55 \pm 9.84 \\
7.96\end{array}$ & $\begin{array}{l}0.08-5.82 \\
1.76 \pm 1.88 \\
1.0\end{array}$ & $25.548^{*}$ & $<0.001 *$ & $<0.001 *$ & $0.010 *$ & $0.014 *$ \\
\hline $\begin{array}{l}\beta \text { globin: } \\
\text { Min.-Max. } \\
\text { Mean } \pm \text { SD. } \\
\text { Median }\end{array}$ & $\begin{array}{l}0.0-1.93 \\
0.34 \pm 0.52 \\
0.10\end{array}$ & $\begin{array}{l}0.0-5.02 \\
1.37 \pm 1.74 \\
0.64\end{array}$ & $\begin{array}{l}0.11-7.25 \\
1.57 \pm 1.83 \\
1.0\end{array}$ & $24.599 *$ & $<0.001 *$ & $0.002 *$ & $<0.001 *$ & 0.067 \\
\hline $\begin{array}{l}\beta / \alpha \text { ratio: } \\
\text { Min.-Max. } \\
\text { Mean } \pm \text { SD. } \\
\text { Median }\end{array}$ & $\begin{array}{l}0.0-2.0 \\
0.32 \pm 0.37 \\
0.20\end{array}$ & $\begin{array}{l}0.0-0.63 \\
0.14 \pm 0.14 \\
0.10\end{array}$ & $\begin{array}{l}0.09-49.61 \\
2.74 \pm 7.76 \\
1.0\end{array}$ & $48.600 *$ & $<0.001 *$ & 0.056 & $<0.001 *$ & $<0.001 *$ \\
\hline $\begin{array}{l}\text { AHSP: } \\
\quad \text { Min.-Max. } \\
\text { Mean } \pm \text { SD. } \\
\text { Median }\end{array}$ & $\begin{array}{l}0.08-87.60 \\
6.89 \pm 14.08 \\
2.91\end{array}$ & $\begin{array}{l}0.07-75.27 \\
32.11 \pm 27.67 \\
39.39\end{array}$ & $\begin{array}{l}0.08-6.13 \\
1.44 \pm 1.70 \\
0.82\end{array}$ & $31.063^{*}$ & $<0.001 *$ & $0.004 *$ & $0.008 *$ & $<0.001 *$ \\
\hline $\begin{array}{l}\alpha / A \text { HSP: } \\
\text { Min.-Max. } \\
\text { Mean } \pm \text { SD. } \\
\text { Median }\end{array}$ & $\begin{array}{l}0.0-1.33 \\
0.20 \pm 0.24 \\
0.15\end{array}$ & $\begin{array}{l}0.01-6.61 \\
0.62 \pm 1.38 \\
0.20\end{array}$ & $\begin{array}{l}0.52-8.18 \\
1.45 \pm 1.23 \\
1.0\end{array}$ & $71.722 *$ & $<0.001 *$ & $0.011 *$ & $<0.001 *$ & $<0.001 *$ \\
\hline $\begin{array}{l}\beta / A H S P: \\
\quad \text { Min.-Max. } \\
\text { Mean } \pm \text { SD. } \\
\text { Median }\end{array}$ & $\begin{array}{l}0.0-0.33 \\
0.05 \pm 0.07 \\
0.02\end{array}$ & $\begin{array}{l}0.0-0.16 \\
0.04 \pm 0.04 \\
0.02\end{array}$ & $\begin{array}{l}0.17-48.12 \\
4.08 \pm 9.99 \\
1.0\end{array}$ & $78.554^{*}$ & $<0.001 *$ & 0.693 & $<0.001 *$ & $<0.001 *$ \\
\hline
\end{tabular}

$\mathrm{K}, p: \mathrm{K}$ and $p$-values for Kruskal Wallis test, Sig. bet. grps was done using Post Hoc Test (Dunn's multiple comparisons test). : Statistically significant at $p \leq 0.05$.

Table (5): Correlation between AHSP expression and different studied parameters.

\begin{tabular}{llllc}
\hline & \multicolumn{4}{c}{ AHSP } \\
\cline { 2 - 5 } & \multicolumn{2}{c}{ Major } & \multicolumn{2}{c}{ Intermedia } \\
\cline { 2 - 5 } & \multicolumn{1}{c}{$r$} & $p$ & $r$ & $p$ \\
\hline Age (years) & -0.269 & 0.094 & -0.277 & 0.084 \\
Sex (male=1, female=2) & -0.141 & 0.386 & -0.136 & 0.401 \\
RBCs (X 10 / c c & -0.151 & 0.351 & 0.216 & 0.181 \\
HB (g/dl) & -0.273 & 0.089 & $0.356^{*}$ & $0.024^{*}$ \\
HCT & -0.165 & 0.308 & $0.331^{*}$ & $0.037^{*}$ \\
MCV & -0.28 & 0.08 & $0.417^{*}$ & $0.007^{*}$ \\
MCH & -0.301 & 0.059 & $0.379^{*}$ & $0.016^{*}$ \\
RDW & 0.246 & 0.126 & 0.109 & 0.504 \\
Pallor & & & & \\
Jaundice & 0.071 & 0.663 & -0.202 & 0.211 \\
Organomegally & $-0.398^{*}$ & $0.011 *$ & -0.279 & 0.082 \\
Growth & 0.054 & 0.74 & - & - \\
Cranio facial changes & -0.286 & 0.074 & & \\
$\alpha$ globin & $0.752^{*}$ & $<0.001 *$ & $0.754^{*}$ & $<0.001 *$ \\
B globin & $0.358^{*}$ & $0.023^{*}$ & $0.749^{*}$ & $<0.001 *$ \\
B globin/ $\alpha$ globin & 0.014 & 0.932 & $0.391 *$ & $0.013^{*}$ \\
HbF\% & 0.14 & 0.391 & -0.114 & 0.486 \\
Ferritin & -0.306 & $0.050^{*}$ & -0.073 & 0.655 \\
Numbers of blood transfusion & $-0.350^{*}$ & $0.027^{*}$ & -0.237 & 0.14 \\
Splenectomy & 0.004 & 0.981 & - & - \\
Severity & - & - & - & - \\
\hline
\end{tabular}

$r$ : Pearson coefficient.

*: Statistically significant at $p \leq 0.05$ 
Table (6): Multivariate linear regression for factors affecting AHSP in thalassemia major group $(n=40)$.

\begin{tabular}{|c|c|c|c|c|c|}
\hline & B & SE & Beta & $t$ & $p$ \\
\hline - Organomegally & -9.819 & 3.596 & -0.268 & 2.731 & $0.010^{*}$ \\
\hline$\bullet \alpha$ globin & 8.051 & 1.171 & 0.906 & 6.878 & $<0.001 *$ \\
\hline$\bullet \beta$ globin & -10.714 & 3.575 & -0.395 & 2.997 & $0.005 *$ \\
\hline $\begin{array}{l}\text { - Numbers of } \\
\text { blood transfusion }\end{array}$ & -0.586 & 0.406 & -0.143 & 1.441 & 0.158 \\
\hline
\end{tabular}

Table (7): Multivariate Linear regression for factors affecting AHSP in thalassemia intermedia group $(n=40)$.

\begin{tabular}{lcclcl}
\hline & B & SE & \multicolumn{1}{c}{ Beta } & \multicolumn{1}{c}{$t$} & \multicolumn{1}{c}{$p$} \\
\hline HB $(\mathrm{g} / \mathrm{dl})$ & -3.628 & 6.125 & -0.191 & 0.592 & 0.558 \\
$\mathrm{HCT}$ & 1.217 & 1.878 & 0.214 & 0.648 & 0.522 \\
$\mathrm{MCV}$ & -1.173 & 0.953 & -0.178 & 1.230 & 0.228 \\
$\mathrm{MCH}$ & 1.398 & 1.863 & 0.096 & 0.751 & 0.458 \\
$\alpha$ globin & 1.780 & 0.292 & 0.633 & $6.092^{*}$ & $<0.001 *$ \\
$\beta$ globin & 6.771 & 2.068 & 0.427 & 3.274 & $0.003 *$ \\
$\beta$ globin/ $\alpha$ globin & 23.611 & 21.634 & 0.122 & 1.091 & 0.283 \\
\hline$r^{2}: 0.810, \mathrm{~F}=19.433, p<0.001 *$ & & &
\end{tabular}

\section{Discussion}

This study aimed to investigate AHSP gene expression in thalassemia major and intermedia patients and to assess its relation with clinical features, hematological parameters alpha and beta globin genes expression and $\mathrm{Hb}$ electrophoresis and serum ferritin to determine its role on the severity of thalassemia. The demographic data showed no significant statistical difference among the studied groups regarding age and sex which accord with [9]. While hematological parameters showed significant difference among groups as regard RBCs, $\mathrm{Hb}, \mathrm{HCT}, \mathrm{MCV}, \mathrm{MCH}$ which were significantly lower in thalassemia major than thalassemia intermedia group. However, RDW was higher in both thalassemic groups versus control group. Anemia in $\beta$ thalassemia results from a combination of ineffective erythropoiesis, peripheral hemolysis, and an overall hemoglobin synthesis diminution [10]. Oxidant injury may result in hemolysis, but there is no proof that it lead to ineffective erythropoiesis [11] . Regarding clinical features of thalassemia, the results revealed that thalassemia major group had severe phenotypic form than thalassemia intermedia group as jaundice, organomegally, growth retardation, craniofacial changes and this comes in agreement with [12]. Increased erythropoietin synthesis enhance formation of extramedullary erythropoietic tissue. Marrow expansion also results in distinctive abnormalities of the skull and face, as well as osteopenia and osteomalacia [13]. Furthermore, Thein et al., correlated the severity of $\beta$-thalassemia with the extent of inequity between $\alpha$ and non- $\alpha$ globin chains and the amount of the free $\alpha$-chain pool. Thus, factors that diminish the extent of chain imbalance and the extent of $\alpha$-chain excess in the red cell precursors will have an impact on the phenotype [10] Interestingly we found out that $\mathrm{HbF} \%$ was higher in both thalassemic groups than control group $(p<0.001)$. Moreover it was higher in thalassemia intermedia group than thalassemia major group $(p<0.001)$. The formation of $\mathrm{HbF} \%$ is the red cell primary compensatory mechanism when there is excess $\alpha$ globin chain for reducing its precipitation which in turn decrease hemolysis in thalassemia intermedia group than thalassemia major group [14]. Moreover thalassemia major group was more frequently blood transfused than thalassemia intermedia group $(p<0.001)$ which agreed with $[15]$ who reported that blood transfusion is an indicator of severity of $\beta$-thalassemia. Consequently serum ferritin was higher in thalassemia major group than thalassemia intermedia group which occurred due to more excessive hemolysis on one hand and more frequent blood transfusion on the other hand. Our results was in accordance with $[\mathbf{9 , 1 4}]$. However ferritin level was higher in thalassemia intermedia group than control group because of increased absorption of dietary iron, as a consequence of ineffective erythropoiesis and rapid turnover of plasma iron [16]. Concerning $\alpha$ globin gene expression, it was higher in thalassemia intermedia compared with thalassemia major and control groups. Also, $\beta$ globin gene expression was markedly lower in thalassemia major compared with thalassemia intermedia and control groups. These results were also reported by Sallaino and his colleagues who depicted that thalassemia intermedia phenotype have excess functional alpha globin genes (alpha gene triplication or quadruplication) which increases the imbalance of the $\alpha /$ non- $\alpha$ globin chain ratio synthesis [17].

Moreover $\beta$ globin $/ \alpha$ globin genes ratio was significantly higher in control group compared to both thalassemia groups, while it did not differ between thalassemia major and intermedia groups. These findings are concordant with $[\mathbf{9 , 1 2}]$, who clarified that the decline in this ratio is mainly because increase alpha chains rather than decrease beta chains formation and added that the main pathophysiological basis of $\beta$ thalassemia is considered to be free $\alpha$ globin chains producing oxidative stress in RBCs and their precursors which in turn induce ineffective erythropoiesis and RBCs hemolysis [18]. Beside [12] reported that $\beta$ globin/ $\alpha$ globin expression ratio was $(0.69 \pm 0.12,0.8 \pm 0.08$ and $0.99 \pm 0.05$ ) in major $\beta$ thalassemia, minor $\beta$ thalassemia and normal controls respectively, which 
indicate decrease ratio in patients in comparison with normal individuals.

The current study depicted that AHSP expression was higher in thalassemia intermedia group versus both major and control groups. Moreover, it was significantly higher in major group versus control group, likewise Ranjbaran et al., reported the same [12]. AHSP help stabilization and solubility of $\alpha$-globin chains to avoid ineffective erythropoiesis by forming a stable but reversible complex with free $\alpha$-globin chains [19-21]. It also prevents toxic effects of $\alpha$-globin by hindering its precipitation and enhancing its thermo-stability [22]. It performs this function by enhancing proper folding of the $\alpha \mathrm{Hb}$, inhibiting auto-oxidation of holo- $\alpha \mathrm{Hb}$, as well as refolding of any denatured protein [21] $\alpha$ globin/AHSP ratio was higher in control group versus both thalassemia groups. Also, thalassemia intermedia group had higher $\alpha$ globin/AHSP versus thalassemia major group, this comes in agreement with Ranjbaran et al., [12]. Who proposed that AHSP expression augment when there is excess $\alpha$ globin expression or increased erythropoietic stress. On the other hand $\beta$ globin/AHSP ratio, was higher in control group versus both thalassemia groups, while it was not significant between both thalassemia groups, this disaccord with Ranjbaran et al., [12]. The diminished amount of this ratio in $\beta$-thalassemia is significantly due to a $\beta$-globin chain decrease but not an AHSP increase. The increased AHSP expression in the situation of $\beta$ globin gene reduction is not sufficient to stop the oppressive effects of extra $\alpha$-globin chain in erythroid cells of patients with severe $\beta$-thalassemia. AHSP can partly recompensate $\beta$-globin deficiency [22,23]. Moreover $\alpha /$ AHSP and $\beta /$ AHSP revealed approximate equal expression of $\beta, \alpha$ and AHSP in normal people inspite of absence of excess free $\alpha$ however AHSP was expressed as much $\alpha$ globin chain this explains that AHSP not only bind free $\alpha$ chains but also to the newely synthesized apo$\alpha$ globin before forming tetramers with $\beta$ globin chains $[12,24]$. The current study showed that AHSP was correlated with $\alpha$ globin and $\beta$ globin gene expression, and also correlated with $\beta$ globin $/ \alpha$ globin ratio in thalassemia intermedia only. This come in agreement with [9]. Moreover data retrieved from the estimation of the $\beta$-globin $/ \alpha$-globin ratio has shown that disease severity increased with a decrease in this parameter. This can authenticate the AHSP molecule function, since AHSP expression levels rise with a diminution of the $\beta / \alpha$ ratio as a compensatory mechanism in $\beta$-globin absence in order to connect the free excess $\alpha$-globin chains and prevent their precipitation in erythroid cells.
Furthermore, Dos santos et al., Mai et al., and Varrichio et al., reported similar results and clarified the importance of AHSP in avoidance of $\alpha$ globin chain precipitation [25-27]. Dos santos et al., showed that AHSP was positively correlated with $\beta$-globin expression and assumed that iron affects AHSP expression as with decrease $\beta$-globin expression, more accessible iron result in destabilization and degradation of AHSP mRNA [26]. With the opportunity that AHSP could be a potential $\beta$ thalassemia modifier, [28] reported that reduced AHSP was correlated with more severe $\beta$ thalassemia phenotype within those with similar $\alpha$ and $\beta$ genotype. Previous studies also reported consistent results where $\beta^{\circ}$ thalassemia erythroblasts had higher AHSP levels than normal erythroblasts $[25,27]$ Moreover, multivariate linear regression revealed that $\alpha$ globin and $\beta$ globin expression are independent factors affecting AHSP gene expression in all thalassemia patients groups, and this emphasize their crucial role in affecting AHSP gene expression. In addition, AHSP was positively correlated with RBCs, Hb, HCT, MCV while negatively correlated with clinical features of thalassemia, ferritin level, frequency of blood transfusions and severity of thalassemia. Astonishingly some previous reports showed no association between AHSP gene expression and severity of symptoms in $\beta$ thalassemia patients $[18,28,29]$. However some investigators identified an effect of AHSP gene expression and its polymorphism on symptoms of $\beta$ thalassemia [26]. This study showed that AHSP gene expression at cutoff $>1$ can discriminate thalassemia cases from healthy control with sensitivity $76.25 \%$, specificity $80 \%$, also, AHSP gene expression at cutoff $\leq 11.31$ can discriminate thalassemia major from thalassemia intermedia with sensitivity $(92.5 \%)$, specificity $(60.0 \%)$. Since the amount of free $\alpha$ globin chain seemed to be an essential factor contributing to differences in hematologic and clinical severity with $\beta$ thalassemia. It was found that AHSP reflect $\beta$ thalassemia severity and its prognosis, so it could potentially offer some therapeutic opportunities in treatment of the complications of $\beta$ thalassemia and other related conditions. Alternatively down regulation of $\alpha$-globin expression was recommended as a potential therapeutical approach using RNA interference, epigenetic drug targeting or genome editing [30]. Finally, we concluded that AHSP expression was higher in thalassemia intermedia group than thalassemia major group. Consequently, AHSP can be considered genetic modifier in $b$ thalassemia due to its ability to reduce globin chain imbalance (chaperon of excess $\alpha$ chain) resulting in milder form of $\beta$ thalassemia. 


\section{Conclusion:}

AHSP expression was higher in thalassemia intermedia group than thalassemia major group. Consequently, AHSP can be considered genetic modifier in 13 thalassemia.

\section{Financial support and sponsorship:}

Nil.

\section{Conflicts of interest:}

There are no conflicts of interest.

\section{References}

1- MURTAZA MUSTAFA, A. THIRU, E.M. IIIZAM, H. FIRDAUS, A.M. SHARIFA, K. FAIRRUL and M.K. NANG: Pathophysiology, Clinical Manifestations, and Carrier Detection in Thalassemia IOSR Journal of Dental and Medical Sciences, 15 (11): 122-6, 2016.

2- METWALLEY K.A. and EL-SAIED A.R.: Glucose homeostasis in Egyptian children and adolescents with [3Thalassemia major: Relationship to oxidative stress. Indian J. Endocrinol. Metab., 18 (3): 333-9, 2014.

3- REES D.C., CLEGG J.B. and WEATHERALL D.J.: Is hemoglobin instability important in the interaction between hemoglobin E and beta thalassemia? Blood, 92 (6): 21416, 1998.

4- SANKARAN V.G. and WEISS M.J.: Anemia: Progress in molecular mechanisms and therapies. Nat. Med., 21 (3): 221-30, 2015.

5- MOLLAN T.L. and ALAYASH A.I.: Redox reactions of hemoglobin: Mechanisms of toxicity and control. Antioxid Redox Signal, 18 (17): 2251-3, 2013.

6- VASSEUR C. and BAUDIN-CREUZA V.: Role of alphahemoglobin molecular chaperone in the hemoglobin formation and clinical expression of some hemoglobinopathies. Transfus. Clin. Biol., 22 (1): 49-57, 2015.

7- SAGAR C.S., KUMAR R., SHARMA D.C. and KISHOR P.: Alpha hemoglobin stabilizing protein: Its causal relationship with the severity of beta thalassemia. Blood Cells Mol. Dis., 55: 104-7, 2015.

8- DORAK M.: Real-time PCR. Clinical Chemistry, 50: 1680-2, 2000.

9- LIM W.F., MUNIANDI L., GEORGE E., SATHAR J., TEH L.K., LAI M.I., et al.: Protein Expression Is In uenced by mean cell haemoglobin and $\mathrm{HbF}$ levels in $\mathrm{HbE} /$ [ 3 thalassaemia individuals. Blood Cells Mol. Dis., 48: 17, 2012.

10- THEIN S.L.: Pathophysiology of $\beta$ Thalassemia-A Guide to Molecular Therapies: Hematology, (1): 3 1-6, 2005.

11- SCHRIER S.L. : Pathophysiology of thalassemia. Curr. Opin. Hematol., 9 (2): 123-6, 2007.

12- RANJBARAN R., OKHOVAT M.A., MOBARHANFARD A., ABOUALIZADEH F., ABBASI M., MOEZZI L., et al.: Relationship between AHSP gene expression, beta/alpha globin mRNA ratio, and clinical severity of the beta-thalassaemia patients, Ann. Clin. Lab. Sci., 44 (2): 189-93, 2014.
13- OLIVIERI N.F. and BRITTENHAM G.M.: Iron chelating therapy and the treatment of thalassemia, Blood, 89: 73961, 1997.

14- THEIN S.L. and MENZEL S.: Discovering the genetics underlying foetal haemoglobin production in adults. $\mathrm{Br}$. J. Haemato., 145: 455-67, 2009.

15- MAHMOUD H.M., SHOEIB A.A., ABD EL-GHANY S.M., REDA M.M. and RAGAB I.A.: Study of alpha hemoglobin stabilizing protein expression in patients with [ 3 thalassemia and sickle cell anemia and its impact on clinical severity Blood Cells Mol. Dis., 55 [4] : 358-62, 2015.

16- AMIT KUMAR MISHRA and ARCHANA TIWARY: Iron Overload in Beta Thalassaemia Major and Intermedia Patients MAEDICA-a Journal of Clinical Medicine, 8 (4): 328-32, 2013.

17- SOLLAINO M.C., PAGLIETTI M.E., PERSEU L., GIAGU N., LOI D. and GALANELLO R.: Association of alpha globin gene quadruplication and heterozygous beta thalassemia in patients with thalassemia intermedia. Haematologica, 94: 1445-8, 2009.

18- VIPRAKASIT V., TANPHAICHITR V.S., CHINCHANG W., SANGKLA P., WEISS M.J. and HIGGS D.R.: Evaluation of alpha hemoglobin stabilizing protein [AHSP] as a genetic modifier in patients with beta thalassemia. Blood, 103: 3296-9, 2004.

19- GELL D., Y. KONG, S.A. EATON, M.J. WEISS and J.P. MACKAY: Biophysical characterization of the alphaglobin binding protein alpha-hemoglobin stabilizing protein. The Journal of biological chemistry, 277: 406029, 2002.

20- KIHM A.J., KONG Y., HONG W., RUSSELL J.E., ROUDA S., ADACHI K., SIMON M.C., BLOBEL G.A. and WEISS M.J.: An abundant erythroid protein that stabilizes free alpha-haemoglobin. Nature, 417: 758-63, 2002.

21- FAVERO M.E. and F.F. COSTA: Alpha-hemoglobinstabilizing protein: An erythroid molecular chaperone. Biochemistry Research International 2011. Volume 2011, Article ID 373859, 7 pages.

22- KONG Y., ZHOU S., KIHM A.J., KATEIN A.M., YU X. GELL, D.A., MACKAY J.P., ADACHI K., FOSTERBROWN L., LOUDEN C.S., GOW A.J. and WEISS M.J.: Loss of alpha hemoglobin stabilizing protein impairs erythropoiesis and exacerbates beta-thalassemia. Journal of Clinical Investigations, 114: 1457-66, 2004.

23- M.J. WEISS and C.O. DOS SANTOS: Chaperoning erythropoiesis, Blood, 113 (10): 2136-44, 2009.

24- YU X., KONG YI., DORE L.C., et al.: “An erythroid chaperone that facilitates folding of $0 c$-globin subunits for hemoglobin synthesis," Journal of Clinical Investigation, 117 (7): 1856-65, 2007.

25- MAI A., JELICIC K., ROTILI D., et al.: Identification of two new synthetic histone deacetylase inhibitors that modulate globin gene expression in erythroid cells from healthy donors and patients with thalassemia, Mol. Pharmacol., 72: 1111-23, 2007.

26- DOS SANTOS C.O., S. ZHOU, R. SECOLIN, X. WANG, A.F. CUNHA, D.R. HIGGS, J.L. KWIATKOWSKI, S.L. THEIN, P.G. GALLAGHER, F.F. COSTA and M.J. 
WEISS.: Population analysis of the alpha hemoglobin stabilizing protein (ahsp) gene identifies sequence variants that alter expression and function. Am. J. Hematol., 83: 103-8, 2008.

27- VARRICCHIO L., FABUCCI M.E., ALFANI E., et al.: Compensated variability in the expressionof globin-related genes in erythroblasts generated ex vivo from different donor, Transfusion, 50: 672-84, 2010.

28- GALANELLO R., PERSEU L., GIAGU N. and SOLE G.: "AHSP expression in 3 -thalassemia carriers with thalassemia intermedia phenotype," Blood, 102: Abstract no. 1881,2003

29- WANG Z., W. YU, Y. LI, X. SHANG, X. ZHANG, F. XIANG and X. XU: Analysis of alpha-hemoglobinstabilizing protein (ahsp) gene as a genetic modifier to the phenotype of beta-thalassemia in Southern China. Blood cells, molecules \& diseases, 45: 128-32, 2010.

30- METTANANDA S., GIBBONS R.J. and HIGGS D.R.: Alpha globin a samolecular target in treatment of betathalassemia. Blood, 125: 3694-701, 2015.

\section{آهمية تعبير البروتين المثبت لهيموجلوبين آلفا في مرضى البيتا ثلاسيميات اليموجيا}

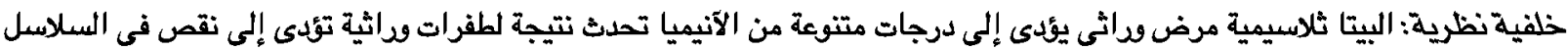

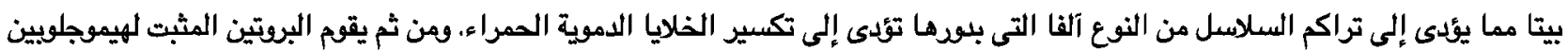

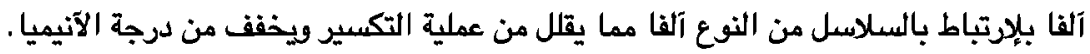

$$
\begin{aligned}
& \text { الهدف من البحث: هو دراسة التعير الجينى اللبرتين المثبت لهيموجلوين آلفا فى مرضى اليتا ثلاسيميا العظمى والوسطى. } \\
& \text { المرضى وطرق البحت: تمت الدراسة على عدد •ع من مرضى البيتا ثلاسيميا العظمى والوسطى وعلى عدد ـع من الآشخاص الآصحاء }
\end{aligned}
$$

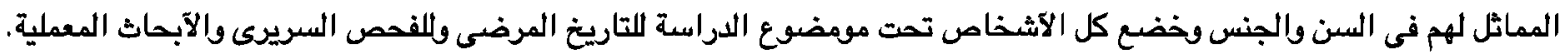

$$
\begin{aligned}
& \text { النتائج: آظهرت النتائج آن التعيير الجينى اللبروتين المثبت لهيموجلوبين آلفا فى مرضى البيتا ثلاسيميا الوسطى آعلى من العظمى ويتناسب } \\
& \text { طرديا هع الهيموجلوبين ونسبة السلاسل من النوع آلفا وبيتا. }
\end{aligned}
$$

الخلاصة: آظهرت النتائج آن التعبير الجينى اللبروتين المثبت لهيموجلويين آلفا فى مرضى الييتا ثلاسيميا الوبطى آعلى من العظمى ولذلك ممكن إعتباره عامل تصسن جينى فى هؤلاء المرضى. 